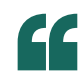

In the opium

poppy,

this bond

formation is

mediated by

an oxidative

enzyme, but

we used simply

the flow of

electrons in

an undivided

electrolysis cell
$\Rightarrow$ BIOMIMETIC TOTAL SYNTHESIS

\section{Coupling aryls at the anode}

Oxycodone is an opioid analgesic produced commercially on a multiton scale. It is widely used for pain management in patients with cancer, but also infamously associated with an epidemic of prescription drug abuse. The commercial production of oxycodone is only semi-synthetic, starting from thebaine - a compound isolated from poppy straw that already contains the core pentacyclic structure. A purely synthetic route to these compounds might eventually help in the search for new structures with improved medicinal properties or that avoid the problems of addiction. Now, writing in Organic Letters, Till Opatz and co-workers from the Johannes Gutenberg University Mainz describe a total synthesis of (-)-oxycodone that relies on an electrochemically driven aryl-aryl coupling.

"During my habilitation, I had developed a method for the synthesis of tetrahydroisoquinoline alkaloids, which are the biogenetic precursors of morphine," says Opatz. "I then began to work with my colleague Siegfried Waldvogel on an anodic coupling approach to form the key aryl-aryl bond." Such an approach would closely resemble the way these compounds are constructed in nature.
Many other chemists have attempted to use oxidative couplings in biomimetic approaches to these structures, but they usually rely on the use of stoichiometric quantities of chemical oxidants. "In the opium poppy, this bond formation is mediated by an oxidative enzyme, but we used simply the flow of electrons in an undivided electrolysis cell with a boron-doped diamond anode to achieve the same result," says Opatz.

The principle of this type of coupling was well established but it had not been applied to the synthesis of morphine alkaloids. The challenge with this approach is that two positions on each aryl ring are activated towards coupling, and thus four possible products can result. Only the $4 \mathrm{a}-2^{\prime}$ coupling (highlighted in the image) can lead to the desired product.

Two developments are important to achieve the desired coupling. The electrochemical procedure is selective for coupling at the $4 \mathrm{a}$ position, in the isoquinoline system. Selective coupling to the 2 ' position, in the phenyl ring, relies on the electronic differentiation provided by the oxygen protecting groups, one acyl and one ether group. Next it is necessary to perform a reactivity screen to identify the best electrochemical conditions.
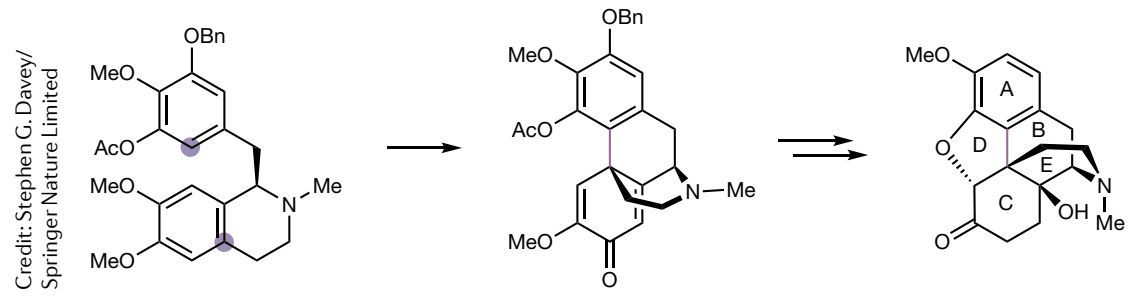

"Screening is mandatory as it is very difficult or even impossible to predict the outcome in a given case," explains Opatz. "Platinum, and boron-doped diamond are universal and highly inert electrode materials, so their use appears logical. On the other hand, the poor performance of a molybdenum anode came as a surprise as this material works nicely for related aryl-aryl couplings."

Formation of the E ring was achieved by cleavage of the acetyl protecting group followed by $\mathrm{S}_{\mathrm{N}} 2^{\prime}$ reaction of the resulting phenol on the reduced quinone ring, affording a diene intermediate. To complete the synthesis, a final hydroxyl group must be added to the $\mathrm{D}$ ring, and the benzyl ether protected oxygen - a remnant of the electronic direction necessary for the selective electrochemical coupling - needs to be excised from the A ring.

The first of these steps was achieved by a photochemically mediated $[4+2]$ cycloaddition of singlet oxygen to the diene, followed by hydrogenolysis of the initially formed endoperoxide. This reduction results in concomitant saturation of the $\mathrm{D}$ ring and cleavage of the benzyl ether. Removal of the superfluous phenolic hydroxyl group was then achieved by formation of a tetrazolyl ether followed by palladium-catalysed hydrogenolysis.

Stephen G. Davey

ORIGINAL ARTICLE Lipp, A. et al. Total synthesis of (-)-oxycodone via anodic aryl-aryl coupling. Org. Lett. https://doi.org/10.1021/acs.orglett. gb00419 (2019)

FURTHER READING Lipp, A. et al. A regio- and diastereoselective anodic aryl-aryl coupling in the biomimetic total synthesis of (-)-thebaine. Angew. Chem. Int. Ed. 57, 11055-11059 (2018) 